\title{
Parameterization of Reactive Force Field for Iron-Water System
}

\author{
Qian CHEN, Jingxiang XU, Yusuke OOTANI, Nobuki OZAWA, Momoji KUBO*
}

Institute for Material Research, Tohoku University, 2-1-1 Katahira, Aoba-ku, Sendai 980-8577, Japan

e-mail:momoji@imr.tohoku.ac.jp

(Received: August 22, 2017; Accepted for publication: October 27, 2017; Online publication: December 2, 2017)

Reactive force field parameters are re-optimized for simulating the stress corrosion cracking (SCC) of ironbased material in a supercritical water environment. The parameters for molecular dynamics (MD) simulation are determined by fitting the adsorption energies of $\mathrm{H}, \mathrm{OH}$, and $\mathrm{H}_{2} \mathrm{O}$ on an $\mathrm{Fe}(110)$ surface obtained by reactive force field to the density functional theory (DFT) calculations. The errors of adsorption energies for the most stable positions are less than 5\%, and our parameters are in good agreement with the DFT calculations. The development of $\mathrm{Fe} / \mathrm{O} / \mathrm{H}$ parameters is expected to contribute to SCC simulation of iron-based materials in supercritical water environments.

Keywords: Molecular dynamics simulation, Reactive force field, DFT calculation, Iron-based material

\section{Introduction}

Advanced ultra-supercritical (A-USC) thermal power plants are expected to offer high efficiency and environmental sustainability. However, stress corrosion cracking (SCC) of the iron-based material widely used in supercritical power stations occurs easily due to chemical reactions between water and the iron-based material, resulting in catastrophic accidents. It is therefore important to understand the chemical reaction dynamics at the iron-water interface in order to improve corrosion resistance. However, experimental observation of chemical reactions at the atomic scale is difficult, particularly in supercritical water environments. Thus, the SCC mechanism in iron-based materials in supercritical water is still unclear. Molecular dynamics (MD) simulation using reactive force field (ReaxFF) is a powerful tool for studying chemical reactions at interfaces [1]. However, to the best of our knowledge, the ReaxFF parameters for describing the interaction between the iron and water have not been well established. In this work, the ReaxFF parameters are re-optimized for the iron-water system in order to investigate the SCC mechanism by MD methods.

\section{Parameterization Method}

To develop ReaxFF parameters for the iron-water system, we calculated the adsorption energies of $\mathrm{H}, \mathrm{OH}$, and $\mathrm{H}_{2} \mathrm{O}$ on an $\mathrm{Fe}(110)$ surface by density functional theory (DFT) using $\mathrm{DMol}^{3}$ code [2,3]. In the DFT calculation, the effective core potentials were used to model the core electron, and PerdewBurke-Ernzerhof generalized gradient approximation was applied to the exchange-correlation term. The Brillouin zone integrations were performed on grids of $3 \times 3 \times 1$ k-points. An energy convergence criterion of $10^{-5}$ Hartree was employed for the geometrical optimization. A previous study reported that $\mathrm{Fe}(110)$ is the most stable among the iron planes [4], and therefore six layers of $\mathrm{Fe}(110)$ surface were used in this work.

\section{Results and Discussion}

To determine the ReaxFF parameters for describing the interaction between iron and water, we first optimized the adsorption structures of $\mathrm{H}$ atom, $\mathrm{OH}$, and $\mathrm{H}_{2} \mathrm{O}$ molecules on the $\mathrm{Fe}(110)$ surface by DFT calculation, and obtained the adsorption energies. In this study, the adsorption on the top, short bridge, long bridge, and hollow sites are calculated (Figure 1). Then, we calculated the potential energies by 


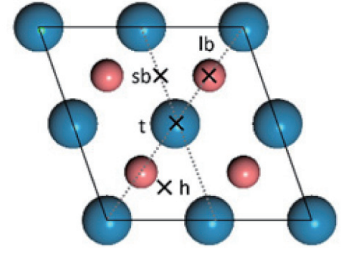

Figure 1. Symmetry sites of top (t), short-bridge (sb), longbridge (lb), and hollow (h) on the Fe(110) surface. Blue and pink balls indicate the surface and subsurface Fe atoms, respectively.

Table 1. Re-optimized parameters for $E_{v a l}$

\begin{tabular}{cccc}
\hline Angle & $\begin{array}{c}\boldsymbol{\Theta}_{\boldsymbol{0}} \\
(\mathrm{deg})\end{array}$ & $\boldsymbol{p}_{\text {val }, 2}(1 / \mathrm{rad})$ & $\boldsymbol{p}_{\text {val }, 4}$ \\
\hline $\mathrm{H}-\mathrm{Fe}-\mathrm{Fe}$ & 15.4956 & 5.0223 & 2.8058 \\
$\mathrm{H}-\mathrm{O}-\mathrm{Fe}$ & 50.9778 & 0.3755 & 0.2323 \\
$\mathrm{Fe}-\mathrm{O}-\mathrm{Fe}$ & 48.6228 & 3.2077 & 0.6779 \\
\hline
\end{tabular}

ReaxFF using the DFT-optimized models to obtain adsorption energies. Finally, we re-optimized the potential parameters of valence angle energy $\left(E_{\mathrm{val}}\right)$ based on previous parameters to fit the DFT calculation results [1]. The re-optimized parameters are presented in Table 1. Here, the meaning of the parameters are explained in the reference [5].

Table 2 shows the adsorption energies of DFT-optimized structures obtained by DFT and ReaxFF calculations. According to DFT calculations, $\mathrm{H}_{2} \mathrm{O}$ has a higher adsorption energy of $-1.696 \mathrm{eV}$ at the top site. The ReaxFF calculation of $\mathrm{H}_{2} \mathrm{O}$ adsorption energies using the parameters we determined shows that the top site is the most stable position with an adsorption energy of $-1.667 \mathrm{eV}$. Thus, our determined parameters successfully describe the adsorption of water molecules on the Fe(110) surface as calculated by DFT. For the adsorption of $\mathrm{H}$, the top and hollow sites are the most stable positions with adsorption an energy of $-0.797 \mathrm{eV}$, and the ReaxFF calculation also gives the largest and second largest adsorption energies of $-0.836 \mathrm{eV}$ at the hollow site and $-0.826 \mathrm{eV}$ at the top site, respectively. In the case of $\mathrm{OH}$ adsorption, the adsorption energies at the hollow and long bridge sites are -4.234 and $-4.227 \mathrm{eV}$, respectively. These values are close, and $\mathrm{OH}$ adsorbs most stably at both the long bridge and hollow sites. ReaxFF calculation shows that the two highest adsorption energies of $\mathrm{OH}$ are $-4.068 \mathrm{eV}$ at the hollow site and $-4.084 \mathrm{eV}$ at the long bridge site, which are also very close. According to the above results, the errors in the adsorption energies between DFT and ReaxFF calculations for the most stable positions are less than 5\%, indicating that our re-optimized parameters are sufficient for describing the interactions between iron and water.

\section{Conclusion}

We re-optimized ReaxFF parameters for investigating the SCC phenomenon of A-USC power plants in supercritical water environments. The adsorption energies of $\mathrm{H}, \mathrm{OH}$, and $\mathrm{H}_{2} \mathrm{O}$ on an $\mathrm{Fe}(110)$ surface calculated by our re-optimized parameters successfully reproduced the DFT results. Thus, simulation based on our developed $\mathrm{Fe} / \mathrm{O} / \mathrm{H}$ parameters is expected to reproduce the interaction between iron and water, and to contribute to investigation of SCC in supercritical water.

\section{Reference}

[1] M. Aryanpour, et al., J. Phys. Chem. A, 114, 6298 (2010). DOI:10.1021/jp101332k

[2] B. Delley, J. Chem. Phys., 92, 508 (1990). DOI:10.1063/1.458452

[3] B. Delley, J. Chem. Phys., 113, 7756 (2000). DOI:10.1063/1.1316015

[4] L. Vitos, et al., Surf. Sci., 411, 186 (1998). DOI:10.1016/ S0039-6028(98)00363-X

[5] A. C. T. van Duin, et al., J. Phys. Chem. A, 105, 9396 (2001). DOI:10.1021/jp004368u

Table 2. Adsorption energies by ReaxFF and DFT calculations.

\begin{tabular}{ccccccc}
\hline & \multicolumn{3}{c}{ DFT $(\mathrm{eV})$} & \multicolumn{3}{c}{ ReaxFF $(\mathrm{eV})$} \\
& $\mathrm{H}$ & $\mathrm{OH}$ & $\mathrm{H}_{2} \mathrm{O}$ & $\mathrm{H}$ & $\mathrm{OH}$ & $\mathrm{H}_{2} \mathrm{O}$ \\
\hline $\mathrm{t}$ & -0.797 & -3.534 & -1.696 & -0.826 & -4.063 & -1.667 \\
$\mathrm{sb}$ & -0.793 & -4.016 & -1.681 & -0.814 & -3.192 & -1.667 \\
$\mathrm{lb}$ & -0.795 & -4.227 & -1.689 & -0.813 & -4.084 & -1.657 \\
$\mathrm{~h}$ & -0.797 & -4.234 & -1.693 & -0.836 & -4.068 & -1.650 \\
\hline
\end{tabular}

